\title{
The effects of sevelamer carbonate on blood glucose and inflammatory factors in patients with diabetic kidney disease; a controlled clinical trial
}

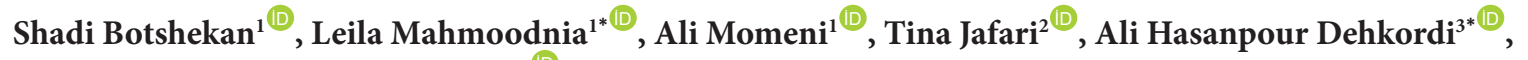 \\ Mohammadsaleh Ghafari Goosheh ${ }^{4}{ }^{\circledR}$ \\ ${ }^{1}$ Department of Internal Medicine, Faculty of Medicine, Shahrekord University of Medical Sciences, Shahrekord, Iran \\ ${ }^{2}$ Nutrition and Dietetics subspecialty, Faculty of Medicine, Shahrekord University of Medical Sciences, Shahrekord, Iran \\ ${ }^{3}$ Associate Professor, Social Determinants of Health Research Center, School of Allied Medical Sciences, Shahrekord University of Medical \\ Sciences, Shahrekord, Iran \\ ${ }^{4}$ Department of Parasitology and Mycology, Faculty of Medicine, Shahrekord University of Medical Sciences, Shahrekord, Iran
}

\section{A R T I C L E I N F O}

\begin{tabular}{l} 
Article Type: \\
Original \\
\hline
\end{tabular}

\section{Article History:}

Received: 11 May 2020

Accepted: 30 June 2020

Published online: 1 August 2020

\section{Keywords:}

Hemoglobin A1c

Inflammatory factors

Diabetic nephropathy

Sevelamer carbonate diabetic

Kidney disease

Chronic kidney disease

End-stage renal disease

\begin{abstract}
A B S T R A C T
Introduction: Diabetic nephropathy (diabetic kidney disease) is a complication of diabetes that begins and progresses with several factors, including hyperglycemia, inflammation and oxidative stress.

Objective: This study demonstrates the effect of sevelamer carbonate administration on hemoglobin Alc levels, blood sugar and inflammatory factors in diabetic nephropathy patients. Patients and Methods: In this clinical trial, 48 diabetic patients under treatment of least one drug who have proteinuria (urinary protein to creatinine ratio greater than 0.5 ) $\geq 2$ times during the past 18 months, received sevelamer carbonate or placebo for two months. Patients' blood samples were taken before and after the intervention. Data were analyzed by SPSS software.

Results: According to the results, fasting blood sugar (FBS), hemoglobin Alc (HbAlc), erythrocyte sedimentation rate (ESR) and C-reactive protein (CRP) showed no significant difference between the study groups before and after the intervention $(P>0.05)$. The mean ICAM-1(intercellular adhesion molecule-1) and VCAM-1 (vascular cell adhesion molecule1) showed no significant difference between the study groups before the intervention $(P>0.05)$. However we found a meaningful difference after the intervention, which was significantly lower in the intervention group $(P<0.05)$.

Conclusion: Sevelamer carbonate administration in patients with diabetic nephropathy has a significant effect on decreasing ICAM-1 and VCAM-1 levels.

Trial registration: The trial protocol was approved in the Iranian Registry of Clinical Trial (identifier: IRCT20191111045401N1; https://en.irct.ir/trial/43662, ethical code; IR.SKUMS. REC.1397.181).
\end{abstract}

Implication for health policy/practice/research/medical education:

Various risk factors such as elevated blood sugar, oxidative stress, elevated concentration of advanced glycosylation end-products and inflammation are involved in diabetic nephropathy. Sevelamer carbonate is a phosphate binding drug that has been proven to have anti-inflammatory and hypoglycemic effects. In our clinical trial, treatment of diabetic nephropathy patients with sevelamer carbonate led to a significant decrease of ICAM-1 (intercellular adhesion molecule-1) and VCAM-1 (vascular cell adhesion molecule1) adhesion molecules which suggests the role of sevelamer carbonate in the recovery of endothelial dysfunction.

Please cite this paper as: Botshekan S, Mahmoodnia L, Momeni A, Hasanpour Dehkordi A, Ghafari Goosheh M. The effects of sevelamer carbonate on blood glucose and inflammatory factors in patients with diabetic kidney disease; a controlled clinical trial. J Nephropharmacol. 2021;10(2):e10. DOI: 10.34172/npj.2021.10.

\section{Introduction}

Diabetic nephropathy (diabetic kidney disease) is one of the serious complications of diabetes which occurs in $30-40 \%$ of diabetic patients. It is the most prevalent cause of chronic kidney disease (CKD), end-stage renal disease (1) and kidney transplantation (2). In addition to causing $\mathrm{CKD}$, diabetic nephropathy increases the rate of death caused by cerebrovascular and peripheral arterial diseases (3). The occurrence and progression of diabetic nephropathy involves multi-factorial risk factors 
including hyperglycemia, inflammation and oxidative stress. Advanced glycation end products (AGEs) play a major role in these risk factors; since they cause both inflammation and oxidative stress (4). AGEs bind to its specific receptor named (RAGE). RAGE-AGEs complex leads to production of several pro-inflammatory cytokines, tumor necrosis factor- $\alpha$ (TNF- $\alpha$ ), interleukin (IL)-6, and pro-fibrotic cytokines such as beta-transforming growth factor. AGEs activate NF- $\kappa$ B transcription factor which leads to expression and release of inflammatory molecules and reactive oxygen species (5). Although hyperglycemia forms AGEs, clinical studies suggest that AGE content of the diet, plays a major role in this area too (6). The obtained data suggests that reduction of AGEs content of diet can be a useful solution for decreasing the complications of diabetes. The finding has been approved by studies in which, using foods containing high levels of AGE has caused insulin resistance, increased inflammation and decreased antioxidant immune system $(6,7)$.

In diabetic kidney disease, there are both inflammatory vascular lesions and glomerulosclerosis, which is manifested by exudative glomerular changes, mesangial sclerosis and hyalinizing alterations in blood vessels. These morphologic lesions gradually decrease glomerular filtration rate during several years (8). Inflammatory cytokines stimulate endothelial cells, then the stimulated endothelium secretes ICAM-1 (intercellular adhesion molecule1) and VCAM-1 (vascular cell adhesion molecules1) (9).

Hyperglycemia increases production of AGE and oxidative stress which causes more adhesion molecules binding to the surface of activated endothelial cells (10). Adhesion molecules that facilitate leukocyteendothelial adhesion and leukocyte infiltration in the diabetic kidneys. Induction of these pathways leads to vascular endothelium dysfunction and increased capillary permeability (8), leading to major damage to small vessels of the glomeruli. It has been revealed that exposure of endothelial cells to uremic milieu leads to expression of monocyte- 1 absorbent protein, IL- 8 , membrane cofactor protein-1 (MCP-1) and VCAM-1. This finding suggests the relationship between vascular damage, systemic inflammation and uremic toxicity (11).

The fundamental treatment for diabetic nephropathy is inhibition of renin-angiotensin-aldosterone system (RAAS) by angiotensin converting enzyme (ACE) inhibitors or angiotensin II receptor inhibitors. Studies suggest that despite improvement of diabetic nephropathy with these drugs, diabetic nephropathy stills occurs and progresses in these patients. Therefore, other mechanisms may be involved in this area (12).

Sevelamer carbonate is a phosphate binder used for controlling serum phosphorus level and preventing hyperphosphatemia in CKD patients undergoing dialysis (13). Clinical studies have suggested that sevelamer carbonate may decrease the serum level of low-molecular weight uremic toxins such as AGEs (14). Sevelamer carbonate has decreased systemic inflammatory response in rat models and hemodialysis patients (15). Furthermore, it can decrease the expression of ICAM-1 and VCAM-1 $(16,17)$. By decreasing hemoglobin A1c (HbA1c), AGEs, inflammation and low-density lipoprotein (LDL-C) risks, sevelamer carbonate can decrease the progression of diabetic nephropathy, albuminuria and deaths related to cardiovascular disease $(18,19)$.

\section{Objectives}

Regarding the blood sugar decreasing effect of sevelamer carbonate and on micro-inflammation, this study aimed to investigate the effect of sevelamer carbonate on $\mathrm{HbAlc}$, blood sugar and inflammatory factors in patients with diabetic nephropathy (diabetic kidney disease).

\section{Patients and Methods \\ Study design}

In the present controlled clinical trial, 48 patients from Shahrekord nephrology clinic with diabetic mellitus with existing proteinuria (with the urinary protein-creatinine ratio of more than 0.5 ) under treatment by at least a drug were included in the study. The inclusion criteria included being affected by diabetic nephropathy, the age of above 18 and informed consent for participation in the study. The exclusion criteria included pregnancy, detected liver disease, active peptic ulcer, using carbamazepine and drug intolerance. After sampling, the patients' information including age, height, weight and body mass index (BMI) were recorded in the questionnaires. The patients were randomly assigned to control and intervention groups based on the random number table. The intervention group received sevelamer carbonate $(1600 \mathrm{mg}$, three times per day, used during fasting) for two months and the control group received placebo tables. Blood sampling was conducted before and after the intervention, since the levels of blood sugar, $\mathrm{HbAlc}$, and inflammatory factors (VCAM-1, ICAM-1, CRP, and ESR) were tested by the ELIZA method in our hospital laboratory (10). The use of aluminum-containing drugs was stopped two months before the study.

\section{Ethical issues}

The research conducted in accordance with the tenets of the Declaration of Helsinki. The Ethics Committee of Shahrekord University of Medical Sciences approved this study (IR.SKUMS.REC.1397.181). Accordingly, written informed consent was taken from all participants before any intervention. The study was extracted from internal medicine residential thesis of Shadi Botshekan at this university. The trial protocol was approved in the Iranian Registry of Clinical Trials (identifier: IRCT20191111045401N1; https://en.irct.ir/trial/43662). 


\section{Statistical analysis}

Data were analyzed using SPSS version 22 software. To describe the data, frequency indices and percentage were used for qualitative variables and mean \pm standard deviation was used for quantitative variables. The normal distribution of variables was evaluated by KolmogorovSmirnov test. To test the differences between the two studied groups, the independent $t$ test (for quantitative normal variables), Kruskal-Wallis $\mathrm{H}$ and Mann-Whitney $\mathrm{U}$ tests (for quantitative abnormal variables) were used. In all tests, the significance level was considered at $P<0.05$.

\section{Results}

In the present study, 48 patients with diabetic nephropathy were studied in the intervention and control groups (Figure 1). The patients' average ages in the control and intervention groups were respectively $67.04 \pm 7.75$ years and $70.79 \pm 9.25$ years $(P>0.05)$. The mean BMI in the intervention group $\left(29.33 \pm 2.32 \mathrm{~kg} / \mathrm{m}^{2}\right)$ was significantly lower than the mean BMI of the control group (30.87 \pm $\left.2.36 \mathrm{~kg} / \mathrm{m}^{2}\right)(P<0.05)$. Therefore BMI was considered as a confounding variable in both groups. However, the analysis did not indicate any difference between the results of the two groups.

The control group included 14 women and 10 men and the intervention group included 11 women and 13 men. There was no significant difference between the two groups in terms of gender $(P>0.05)$. According to Table
1, the patients' mean HbA1c, FBS and ESR in two groups were not significantly different before the intervention $(P>0.05)$; however, the results were significantly different after the intervention while the intervention group reported lower means than the control group $(P>0.05)$. According to Tables 2 and 3, before the intervention, two subjects of the control group and three subjects of the intervention group reported positive CRP $(\geq 1 \mathrm{mg} / \mathrm{L})$; whereas after the intervention, two subjects of each group reported positive CRP.

\section{Discussion}

In the present study, 48 patients with diabetic nephropathy were treated in the intervention (sevelamer carbonate) and control (placebo) groups for 8 weeks. We found no significant difference between HbAlc and FBS in the two groups before and after the intervention. Yubero-Serrano et al in a clinical trial on diabetic patients (with HbAlc of above 6.5\%) with $\mathrm{CKD}$ (stages 1-4) reported that receiving $1600 \mathrm{mg}$ sevelamer carbonate for 8 weeks leads to a significant decrease of AGEs without any significant change in $\mathrm{HbAlc}$ or albumin-creatinine ratio. Likewise, the significant decreases of $\mathrm{HbA} 1 \mathrm{c}$ and albumin-creatinine ratio were respectively reported in women and people below 65 years who had diabetic kidney disease through the study of Yubero-Serrano et al (17). Vlassara et al reported that prescribing sevelamer carbonate for diabetic patients with CKD leads to a significant decrease of serum AGE

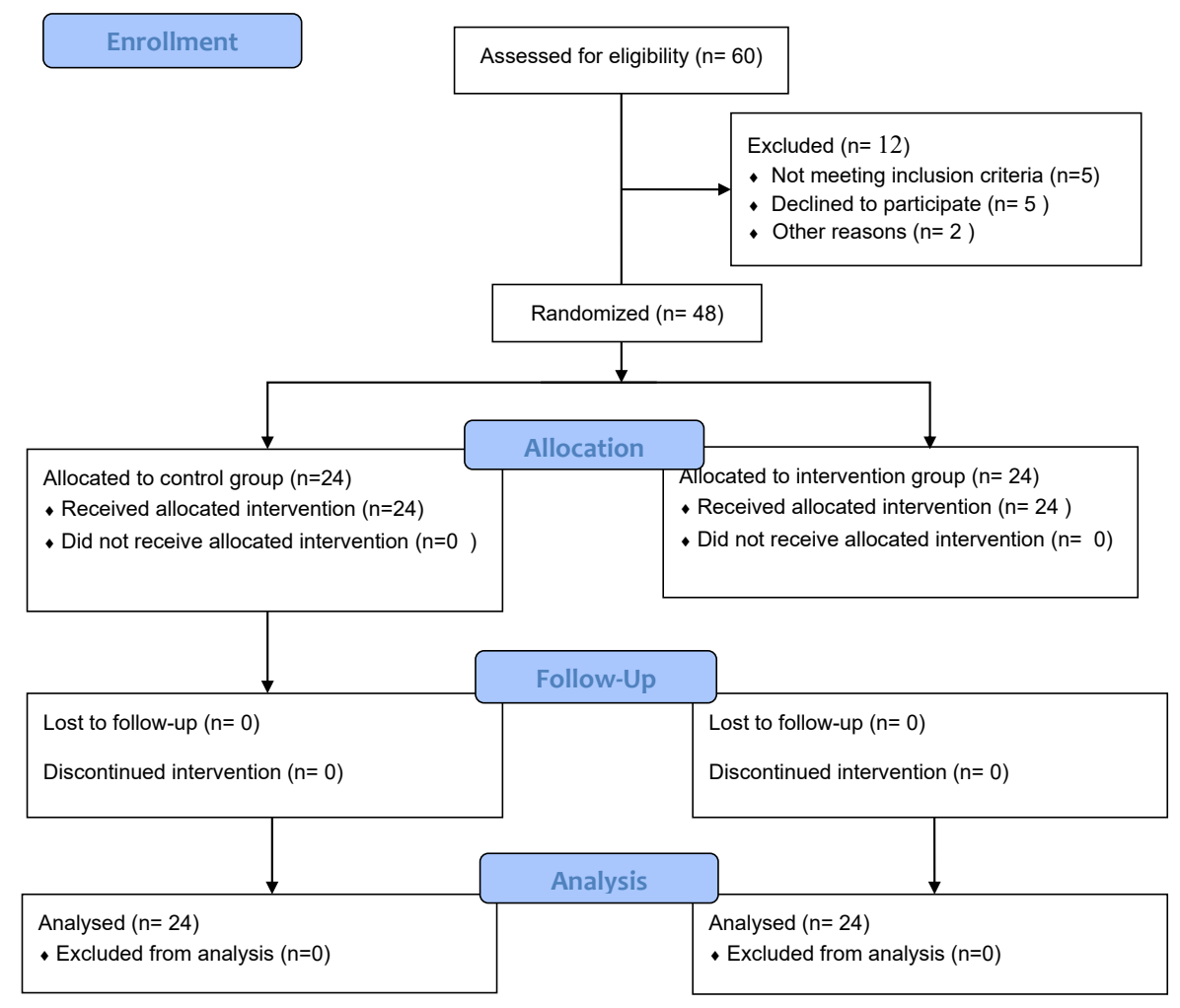

Figure 1. Consort flowchart of the study. 
Table 1. Comparison of $\mathrm{HbA} 1 \mathrm{c}, \mathrm{FBS}$, and inflammatory factors in the studied groups before and after the intervention

\begin{tabular}{|c|c|c|c|c|}
\hline Variable & Level & $\begin{array}{c}\text { Control } \\
\text { Mean } \pm \text { SD }\end{array}$ & $\begin{array}{c}\text { Intervention } \\
\text { Mean } \pm \text { SD }\end{array}$ & $P$ value \\
\hline \multirow{2}{*}{$\mathrm{FBS}(\mathrm{mg} / \mathrm{dL})$} & Before the intervention & $166.41 \pm 46.27$ & $170.08 \pm 35.29$ & 0.75 \\
\hline & After the intervention & $167.33 \pm 39.63$ & $151.75 \pm 31.57$ & 0.139 \\
\hline \multirow{2}{*}{$\mathrm{HbA} 1 \mathrm{c} \%$} & Before the intervention & $8.50 \pm 1.64$ & $8.27 \pm 1.15$ & 0.579 \\
\hline & After the intervention & $8.60 \pm 1.88$ & $7.81 \pm 1.28$ & 0.098 \\
\hline \multirow{2}{*}{$\mathrm{ESR}(\mathrm{mm} / \mathrm{h})$} & Before the intervention & $19.62 \pm 13.81$ & $20.00 \pm 12.02$ & 0.921 \\
\hline & After the intervention & $17.87 \pm 10.31$ & $15.41 \pm 10.31$ & 0.413 \\
\hline \multirow{2}{*}{ ICAM-1 ( ng/L) } & Before the intervention & $396.12 \pm 176.91$ & $360.26 \pm 193.93$ & 0.507 \\
\hline & After the intervention & $393.92 \pm 166.95$ & $287.15 \pm 204.44$ & $0.054^{*}$ \\
\hline \multirow{2}{*}{ VCAM-1 ( $\mathrm{ng} / \mathrm{L})$} & After the intervention & $21.75 \pm 25.09$ & $21.09 \pm 26.39$ & 0.063 \\
\hline & After the intervention & $20.52 \pm 17.19$ & $17.93 \pm 27.29$ & $0.011^{*}$ \\
\hline
\end{tabular}

Table 2. Comparison of CRP distribution in the studied groups before the intervention

\begin{tabular}{|c|c|c|c|c|c|c|c|c|c|c|}
\hline \multirow{2}{*}{ Group } & \multicolumn{10}{|c|}{$\mathrm{CRP}(\mathrm{mg} / \mathrm{L})$} \\
\hline & 2 & 3 & 4 & 5 & 6 & 7 & 8 & 10 & 12 & Total \\
\hline \multirow{2}{*}{ Control } & 5 & 7 & 3 & 3 & 4 & 1 & 1 & 0 & 0 & 24 \\
\hline & 20.8 & 29.2 & 12.5 & 12.5 & 16.7 & 4.2 & 4.2 & 0 & 0 & 100 \\
\hline \multirow{2}{*}{ Intervention } & 3 & 10 & 3 & 4 & 1 & 1 & 0 & 1 & 1 & 24 \\
\hline & 12.5 & 41.7 & 12.5 & 16.7 & 4.2 & 4.2 & 0 & 4.2 & 4.2 & 100 \\
\hline
\end{tabular}

Table 3. Comparison of CRP distribution in the studied groups after the intervention

\begin{tabular}{|c|c|c|c|c|c|c|c|c|}
\hline \multirow{2}{*}{ Group } & \multicolumn{8}{|c|}{$\mathrm{CRP}(\mathrm{mg} / \mathrm{L})$} \\
\hline & 0 & 2 & 3 & 4 & 5 & 6 & 7 & Tota \\
\hline \multirow{2}{*}{ Control } & 12 & 1 & 2 & 1 & 1 & 5 & 2 & 24 \\
\hline & 50 & 4.2 & 8.3 & 4.2 & 4.2 & 20.8 & 8.3 & 100 \\
\hline \multirow{2}{*}{ Intervention } & 13 & 1 & \multirow{2}{*}{312.5} & 0 & 2 & 3 & 2 & 24 \\
\hline & 54.2 & 4.2 & & 0 & 8.3 & 12.5 & 8.3 & 100 \\
\hline
\end{tabular}

and HbA1c (10). Recent findings showed that sevelamer carbonate can improve glucose metabolism by decreasing AGEs. Additionally, recent studies have reported that decreased AGEs are accompanied by increased insulin sensitivity and improved glucose metabolism (7).

In the present study, no significant difference between mean ESR and positive CRP cases in the two groups before and after the intervention was seen. Before the intervention, two subjects of the control group and three subjects of the intervention group reported positive CRP $(\geq 6 \mathrm{mg} / \mathrm{L})$; whereas after the intervention, two subjects of each group reported positive CRP. Yamada et al reported that prescription of sevelamer carbonate for hemodialysis patients leads to a significant decrease of high sensitive CRP (hs-CRP) serum levels after 12 and 24 weeks (20). Previously Stinghen et al reported that administration of sevelamer carbonate for hemodialysis patients for one month leads to a significant decrease of hs-CRP compared to the control group (21). Vlassara et al reported that administration of sevelamer carbonate for patients with diabetic nephropathy for two months leads to a significant decrease of inflammatory markers like
TNF-a (10). Accordingly, Chennasamudram et al found that prescription of sevelamer carbonate for patients undergoing peritoneal dialysis for eight weeks led to a significant decrease of CRP levels (22). Shantouf et al performed a study to investigate the effects of sevelamer carbonate and calcium binder on inflammatory markers and lipids in hemodialysis patients. They found that sevelamer carbonate is a reliable treatment for hemodialysis patients with a high risk of cardiovascular diseases, while it significantly decreases LDL-c and inflammatory markers such as CRP (18). In our study, the low effect of sevelamer carbonate on CRP and ESR can be due to the few number of positive CRP cases and the shorter period of intervention compared to the above mentioned studies. In general, CRP is not a reliable inflammation factor and it is affected by various conditions. Furthermore, no certain normal range has been reported for that, however, CRP serum level of above $6 \mathrm{mg} / \mathrm{L}$ is an indicator of inflammation (23). In order to confirm the anti-inflammatory effects of sevelamer carbonate in patients with nephropathy, it is suggested to use other inflammation markers such as TNF-a too. 
In this study, no significant difference between the means of ICAM-1 and VCAM-1 in two groups before the intervention was detected. However a significant difference was reported after the intervention while the intervention group had a significantly lower acute phase reactant result. Several studies have investigated the effect of sevelamer carbonate on endothelial function. Gregorio et al found that exposure of endothelial cells to AGEs increases the expression of RAGE and inflammatory biomarkers and activates endothelial factors, PAIplasminogen activator inhibitor-1, MCP-1, IL-8, IL-6, serum amyloid A and monocyte chemotoxic factor, while treatment by sevelamer carbonate recovers the mentioned factors to the normal levels. In fact the results suggested that sevelamer carbonate decreases the expression of RAGE and the markers of endothelial dysfunction caused by AGEs in uremic environment (5). Animal studies have indicated that sevelamer carbonate can decrease the expression of adhesion molecules including ICAM-1 and VCAM-1 as the vascular damage markers and cell and serum AGE levels.

Sevelamer carbonate also can strengthen antioxidant defense and decrease circulating peroxidation levels $(16,24)$. A study investigating CKD patients showed that eight weeks of sevelamer carbonate treatment can improve endothelial function by significantly increasing flow mediated dilation (from $6.1 \%$ to $7.1 \%$ ) and decreasing fibroblast growth factor-23 (25). In the present study, treatment of patients with diabetic nephropathy with sevelamer carbonate led to a significant decrease of ICAM-1 and VCAM-1 adhesion molecules which suggests the role of sevelamer carbonate in recovery of endothelial dysfunction.

\section{Conclusion}

According to the results, treatment of diabetic nephropathy patients with sevelamer carbonate led to a significant decrease of ICAM-1 and VCAM-1 adhesion molecules which suggests the role of sevelamer carbonate in the recovery of endothelial dysfunction. Sevelamer carbonate decreased ESR as an inflammation marker, FBS and $\mathrm{HbAlc}$ as glucose metabolism markers. However, the difference with the control group was not significant. Therefore, sevelamer carbonate can be used as an effective drug for recovery of endothelial dysfunction and prevention of nephropathy progression. However, further studies should be performed to investigate its other effects, acceptance and the probable side effects.

\section{Limitations of the study}

Possibility of complications for patients who were identified and controlled with repeated follow-up

\section{Authors' contribution}

SB, LM, TJ, MGG and AH were the principal investigators of the study and they were included in preparing the concept and designing of the study. SB, LM and AM were involved in performing study tests and data acquiring. LM and SB revised the manuscript. All authors participated in preparing the final draft of the manuscript.

\section{Conflicts of interest}

The authors declare that they have no competing interests.

\section{Ethical considerations}

Ethical issues (including plagiarism, data fabrication, double publication) have been completely observed by the authors.

\section{Funding/Support}

The study was extracted from the internal medicine residential thesis of Shadi Botshekan at Shahrekord University of Medical Sciences, which supported by the university.

\section{References}

1. Warren AM, Knudsen ST, Cooper ME. Diabetic nephropathy: an insight into molecular mechanisms and emerging therapies. Expert Opin Ther Targets. 2019;23:57991. doi: 10.1080/14728222.2019.1624721. E

2. Dousdampanis P, Trigka K, Mouzaki A. Tregs and kidney: From diabetic nephropathy to renal transplantation. World J Transplant 2016;6:556. doi: 10.5500/wjt.v6.i3.556

3. Buchberger B, Follmann M, Freyer D, Huppertz H, Ehm A, Wasem J. The importance of growth factors for the treatment of chronic wounds in the case of diabetic foot ulcers. GMS Health Technol Assess. 2010;6:158-63. doi: 10.3205/hta000090

4. Makita Z, Radoff S, Rayfield EJ, Yang Z, Skolnik E, Delaney $\mathrm{V}$, et al. Advanced glycosylation end products in patients with diabetic nephropathy. N E J Med. 1991;325:836-42. doi: 10.1056/NEJM199109193251202

5. Gregório P, Favretto G, Sassaki G, Cunha R, BeckerFinco A, Pecoits-Filho R. Sevelamer reduces endothelial inflammatory response to advanced glycation end products. Clin Kidney J. 2017;3:1-10. doi: 10.1093/ckj/sfx074

6. Koschinsky T, He C-J, Mitsuhashi T, Bucala R, Liu C, Buenting $\mathrm{C}$, et al. Orally absorbed reactive glycation products (glycotoxins): an environmental risk factor in diabetic nephropathy. Proceed Nation Acad Sci. 1997;94:6474-9. doi: 10.1073/pnas.94.12.6474

7. Uribarri J, Cai W, Ramdas M, Goodman S, Pyzik R, Chen $\mathrm{X}$. Restriction of advanced glycation end products improves insulin resistance in human type 2 diabetes. Diabetes Care. 2011;7:1610-6. doi: 10.2337/dc11-0091

8. Hocaoglu-Emre FS, Saribal D, Yenmis G, Guvenen G. Vascular cell adhesion molecule 1, intercellular adhesion molecule 1, and cluster of differentiation 146 levels in patients with Type 2 diabetes with complications. Endocrinol Metabol. 2017;32:99-105. doi: 10.3803/ EnM.2017.32.1.99

9. Hadi HA, Al Suwaidi J. Endothelial dysfunction in diabetes mellitus. Vasc Health Risk Manag. 2007;3:853. 
10. Vlassara H, Uribarri J, Cai W, Goodman S, Pyzik R, Post $\mathrm{J}$, et al. Effects of sevelamer on $\mathrm{HbA1c}$, inflammation, and advanced glycation end products in diabetic kidney disease. Clin J Am Soci Nephrol. 2012;7:934-42. doi: 10.2215/ CJN.12891211.

11. Stinghen A, Goncalves S, Martines E, Nakao L, Riella M, Aita C. Increased plasma and endothelial cell expression of chemokines and adhesion molecules in chronic kidney disease. Nephron Clin Pract 2009;111:c117. doi: $10.1159 / 000191205$

12. Nguyen G, Delarue F, Burcklé C, Bouzhir L, Giller T, Sraer J-D. Pivotal role of the renin/prorenin receptor in angiotensin II production and cellular responses to renin. J Clin Invest. 2002;109:1417-27.

13. Zhou T, Li H, Xie W, Lin Z. A meta-analysis of phosphate binders lanthanum carbonate versus sevelamer hydrochloride in patients with end-stage renal disease undergoing hemodialysis. Afr Health Sci. 2018;18:689-696. doi: 10.4314/ahs.v18i3.27.

14. Garg J, Chasan-Taber S, Blair A P, M, Bommer J, Raggi P. Effects of sevelamer and calcium-based phosphate binders on uric acid concentrations in patients undergoing hemodialysis: A randomized clinical trial. Arthritis Rheumatol. 2005;52:290-5. doi: 10.1002/art.20781.

15. Hauser A, Azevedo I, Gonçalves S, Stinghen A, Aita C, Pecoits-Filho R. Sevelamer carbonate reduces inflammation and endotoxemia in an animal model of uremia. Blood Purif. 2010;30:153-8. doi:10.1159/000319850.

16. Maizel J, Six I, Dupont S, Secq E, Dehedin B, Barreto F. Effects of sevelamer treatment on cardiovascular abnormalities in mice with chronic renal failure. Kidney Int. 2013;84:491-500. doi:10.1038/ki.2013.110.

17. Yubero-Serrano E, Woodward M, Poretsky L, Vlassara H, Striker G. Effects of sevelamer carbonate on advanced glycation end products and antioxidant/pro-oxidant status in patients with diabetic kidney disease. Clin J Am Soc Nephrol. 2015;10:759-66. doi: 10.2215/CJN.07750814

18. Shantouf R, Budoff M, Ahmadi N, Tiano J, Flores F,
Kalantar-Zadeh K. Effects of sevelamer and calcium-based phosphate binders on lipid and inflammatory markers in hemodialysis patients. Am J Nephrol. 2008;28:275-9. doi:10.1159/000111061.

19. Raggi P, Vukicevic S, Moysés R, Wesseling K, Spiegel D. Ten-year experience with sevelamer and calcium salts as phosphate binders. Clin J Am Soc Nephrol. 2010;5:31-40. doi: $10.2215 /$ CJN.05880809.

20. Yamada K, Fujimoto S, Tokura T, Fukudome K, Ochiai $\mathrm{H}$, Komatsu $\mathrm{H}$, et al. Effect of sevelamer on dyslipidemia and chronic inflammation in maintenance hemodialysis patients. Renal Fail. 2005;27:361-5. doi:10.1081/JDI-65259.

21. Stinghen A, Gonçalves S, Bucharles S, Branco F, Gruber B, Hauser A. Sevelamer decreases systemic inflammation in parallel to a reduction in endotoxemia. Blood Purif. 2010;29:352-6. doi: 10.1159/000302723.

22. Chennasamudram SP, Noor T, Vasylyeva TL. Comparison of sevelamer and calcium carbonate on endothelial function and inflammation in patients on peritoneal dialysis. J Renal Care. 2013;39: 82-9. doi: 10.1111/j.1755-6686.2013.12009.x.

23. Caballero A, Delgado A, Aguilar-Salinas C, Herrera A, Castillo J, Cabrera T, et al. The differential effects of metformin on markers of endothelial activation and inflammation in subjects with impaired glucose tolerance: a placebo-controlled, randomized clinical trial. J Clin Endocrinol Metab 2004;89:3943-8. doi: 10.1210/jc.20040019.

24. LengletA,FabresseN, TaupinM, GomilaC,LiabeufS, KamelS, Does the Administration of Sevelamer or Nicotinamide Modify Uremic Toxins or Endotoxemia in Chronic Hemodialysis Patients? Drugs. 2019;79:855-862. doi: 10.1007/s40265-019-01118-9.

25. Yilmaz MI, Sonmez A, Saglam M, Yaman H, Kilic S, Eyileten $\mathrm{T}$, et al. Comparison of calcium acetate and sevelamer on vascular function and fibroblast growth factor 23 in CKD patients: a randomized clinical trial. Am J Kidney Dis. 2012;59:177-85. doi: 10.1053/j.ajkd.2011.11.007.

Copyright $\odot 2021$ The Author(s); Published by Published by Society of Diabetic Nephropathy Prevention. This is an open-access article distributed under the terms of the Creative Commons Attribution License (http://creativecommons.org/licenses/by/4.0), which permits unrestricted use, distribution, and reproduction in any medium, provided the original work is properly cited. 